\title{
Physical Effects of Extra Dimension and Concomitant Map between Photons and Gravitons in Randall-Sundrum Brane-World Scenario
}

\author{
Roldão da Rocha*† \\ Instituto de Física Teórica, Universidade Estadual Paulista, Rua Pamplona 145 \\ 01405-900 São Paulo, SP, Brazil \\ and \\ DRCC - Instituto de Física Gleb Wataghin, Universidade Estadual de Campinas, CP 6165, \\ 13083-970 Campinas, SP, Brazil \\ E-mail: roldaodifi.unicamp.br, roldaodift.unesp.br

\section{Carlos H. Coimbra-Araújo} \\ Instituto de Física Gleb Wataghin, Universidade Estadual de Campinas, CP 6165 13083-970 \\ Campinas, SP, Brazil. \\ E-mail: carlosceifi.unicamp.br
}

\begin{abstract}
We investigate, in Randall-Sundrum braneworld scenario, the relationship between perturbations of gravitational and electromagnetic waves in a black hole neighboorhood, proposing an extradimensional braneworld extension of Novikov's formalism. Mutual transformations of electromagnetic and gravitational fields due to the strong fields in Reissner-Nordstrøm black holes are analyzed from an effective 5-dimensional Randall-Sundrum perspective.
\end{abstract}

Fifth International Conference on Mathematical Methods in Physics

24 - 28, April 2006

Rio de Janeiro, Brazil

\footnotetext{
* Speaker.

${ }^{\dagger}$ Thanks to FAPESP for financial support
} 


\section{Introduction}

The possibility concerning the existence of extra dimensions is one of the most astonishing aspects of string theory and the formalism of p-branes. In spite of this possibility, extra dimensions still remain up to now unaccessible and obliterated to experiments. An alternative approach to the compactification of extra dimensions, provided by, e.g., Kaluza-Klein (KK) and string theories $[1,2,3,4]$, involves an extra dimension which is not compactified, as pointed by Randall-Sundrum model $[5,6]$. This extra dimension implies deviations on Newton's law of gravity at scales below about $0.1 \mathrm{~mm}$, where objects may be indeed gravitating in more dimensions. The electromagnetic, weak and strong forces, as well as all the matter in the universe, would be trapped on a brane with three spatial dimensions, and only gravitons would be allowed to leave the surface and move into the full bulk, constituted by an $\mathrm{AdS}_{5}$ spacetime, as prescribed by in Randall-Sundrum model $[5,6]$.

Certainly black holes (BHs) are among the most fascinating and counterintuitive objects predicted by theoretical physics. One of the astonishing features concerning BHs comes from the effects produced by the gravitational extreme limit associated with these objects, as the bending of light, the redshifting of clocks or high energy astrophysical phenomena. An interesting claimed effect occurs at the neighborhood of BHs; it seems that standard 4D gravitational waves (GWs) can interact with electric and magnetic fields to produce electromagnetic (EM) waves [8, 9, 10, 11, 12]. For a detailed complete list see [13]. This is described as a mutual transformation of electromagnetic and gravitational fields due to the strong fields present in the neighborhood of some BHs [7], and it is a well known corollary of the non-linearity of Einstein-Maxwell equations. For instance, when a plane gravity wave passes through a magnetic field, it vibrates the magnetic field lines, thus creating EM radiation [12]. In particular we are interested to focus this interesting aspect in the context of extra-dimensional braneworld scenarios [14] (for a review see [15]). The evolution of gravitational wave perturbations in braneworld Randall-Sundrum cosmology have been extensively investigated in [16], where it was demonstrated the zero mode of the 5-dimensional graviton is generated, while the massive modes remain in their vacuum state.

The main aim of this paper is to present an alternative way to observe extra-dimensional signatures present in the electromagnetic spectrum of some galaxies. A particular proposal to accomplish it, using the Randall-Sundrum model, was proposed by [18, 19]. Here we will use a brane-corrected Einstein-Maxwell equation to derive an useful system of gauge covariant equations proposed by [20], and we assume the possibility of GWs modes generated by perturbations in extra-dimensional sections of a Reissner-Nordstrøm BH. Extending Novikov's result, we calculate the braneworld corrections concerning Einstein-Maxwell equations. GWs modes interact with the electromagnetic field of a BH to produce EM waves. Recent calculations using braneworld scenarios [21] show that most massive BHs can possess an extended extra-dimensional tail - the black string. Perturbations on the black string - e.g. caused by mergers among BHs — produce vibrating modes known as Kaluza-Klein (KK) modes, capable to propagate in our spacetime due to the geometrical projection of extra dimensions in 3D space. This paper is organized as follows: in Sec. 2 we solve Eisntein-Maxwell equations on the brane in the neighborhood of a Reisnerr-Nordstrøm BH, using an eikonal approximation involving a null tetrad, and we prove that in the Randall-Sundrum model there are more terms due to braneworld effects, involving only the amplitude of the perturbation in the EM potential, and the amplitude of perturbation in the metric that endows the 3-brane. We also 
discuss our results and the graphics obtained in Concluding Remarks.

\section{Solutions of Einstein-Maxwell equations on the brane}

It is possible to project extra dimensions in the 3D brane space and generate corrections in Einstein field equations by a Gauss-Codazzi extrinsic curvature projection treatment [22, 23]. The associated corrected Einstein equations induced on the brane, assuming $\mathbb{Z}_{2}$-simmetry, Israel-Darmois junction conditions and the Bianchi identities, read [15, 19]

$$
G_{\mu v}=-\Lambda g_{\mu v}+\kappa^{2} T_{\mu v}+6 \frac{\kappa^{2}}{\lambda} \mathscr{S}_{\mu v}-\mathscr{E}_{\mu \nu}
$$

where $S_{\mu \nu}$ is given by $[15,19]$

$$
\mathscr{S}_{\mu v}=\frac{1}{12} T T_{\mu v}-\frac{1}{4} T_{\mu \alpha} T_{v}^{\alpha}+\frac{1}{24} g_{\mu v}\left(3 T_{\alpha \beta} T^{\alpha \beta}-T^{2}\right)
$$

where $T^{2}=\left(T_{\alpha}{ }^{\alpha}\right)^{2}$. The term $\mathscr{E}_{\mu \nu}$ is the projection of the Weyl tensor on the brane and $\Lambda$ denotes the cosmological constant.

Denoting $h_{\mu v}=\delta g_{\mu v}$ and $h=h_{\alpha}^{\alpha}$, from the perturbations of the Christoffel symbols given by $\delta \Gamma_{\mu \nu}^{k}=\frac{1}{2}\left(h^{k}{ }_{v ; \mu}-h_{\mu ; \nu}^{k}-h_{\mu \nu}^{; k}\right)$ and denoting $k_{\mu v}=h_{\mu v}-\frac{1}{2} h g_{\mu v}$, it follows that the variation of Eq.(2.1) gives

$$
k_{\mu v ; \lambda}^{; \lambda}-k_{\mu ; v ; \lambda}^{\lambda}-k_{v ; \mu ; \lambda}^{\lambda}-\frac{1}{2} g_{\mu v} k_{; \lambda}^{; \lambda}-6 \frac{\kappa^{2}}{\lambda} \delta \mathscr{S}_{\mu v}+\delta \mathscr{E}_{\mu v}-\kappa^{2} \delta T_{\mu v}=0
$$

where

$$
\begin{aligned}
\delta \mathscr{S}_{\mu v}= & \left.-g_{\mu v}\left(4 \delta F_{a m} F_{b}^{m} F^{a c} F_{c}{ }^{b}-F^{2}\left(2 \delta F_{a b} F^{a b}-F_{a \alpha} F_{b}{ }^{\alpha} h^{b a}\right)+\frac{1-F^{2}}{2} h^{m n} F_{m a} F_{n}{ }^{a}+4 h^{m n} F_{c}^{a} F_{b}^{c} F_{a m} F_{n}^{b}\right)\right] \\
& -g_{\mu v} \frac{h\left(F^{2}\right)^{2}}{4}-\frac{\left(F^{2}\right)^{2}}{2} h_{\mu v}-\frac{h}{2} F^{2} F_{\mu \alpha} F_{v}{ }^{\alpha}+\frac{F^{2}}{2} \delta F_{m(v} F_{\mu)}{ }^{m}+\delta F_{a m} F^{\alpha a} F_{\alpha(\mu} F_{v)}{ }^{m}-F^{\alpha a} F_{a}{ }^{m} \delta F_{m(\mu} F_{v) \alpha} \\
& +\frac{1}{2} \delta F_{m n} F^{m n} F_{\alpha(v} F_{\mu)}^{\alpha}+h^{m n}\left(F_{m}^{a} F_{a}^{\alpha} F_{n(v)} F_{\mu) \alpha}+2 F_{m a} F_{n}{ }^{a} F_{\alpha(\mu} F_{v)}{ }^{\alpha}\right)-\frac{F^{2}}{4} F_{a}^{\alpha} h^{a}{ }_{(v} F_{\mu) \alpha},
\end{aligned}
$$

$F^{2}=\left(F_{\alpha}{ }^{\alpha}\right)^{2}$, and the variation of electro-vacuum momentum-energy tensor $T_{\mu v}=\left(F_{\sigma \mu} F_{v}^{\sigma}-\right.$ $\left.\frac{1}{4} g_{\mu \nu} F^{2}\right) / 4 \pi$ is given by

$$
\begin{array}{r}
4 \pi \delta T_{\mu v}=\delta F_{\mu \alpha} F_{v}^{\alpha}+F_{\mu \alpha} \delta F_{v}^{\alpha}+F_{\mu \alpha} F_{v}^{\beta} h_{\beta}{ }^{\alpha}-\frac{1}{4} h_{\mu v} F^{2}-\frac{1}{2} g_{\mu v} \delta F_{\alpha \beta} F^{\alpha \beta} \\
-\frac{1}{2} g_{\mu v} h_{\gamma}{ }^{\alpha} F_{\alpha \beta} F^{\gamma \beta}-\frac{1}{2} g_{\mu v} h^{\alpha \beta} F_{\alpha \rho} F_{\beta}{ }^{\rho} .
\end{array}
$$

Variation of Maxwell equations is written as

$$
\delta F_{; \beta}^{\alpha \beta}-k_{\beta \rho} F^{\alpha \rho ; \beta}-k_{\mu ; \beta}^{\alpha} F^{\mu \beta}-k_{\mu \beta}^{; \beta} F^{\alpha \mu}+\frac{1}{2} k_{, \beta} F^{\alpha \beta}=0
$$

Besides, given a local chart $\left\{x^{\sigma}\right\}$ on the brane, there are electromagnetic 1-form field potentials $A=A_{\mu} d x^{\mu}$ and $\mathfrak{a}=\mathfrak{a}_{\rho} d x^{\rho}$, respectively associated with the electromagnetic 2-form field $F=$ $F_{\mu v} d x^{\mu} \wedge d x^{v}$ and its variation $\delta F$, whose components are related by

$$
F_{\mu v}=A_{v, \mu}-A_{\mu, v}, \quad \delta F_{\mu v}=\mathfrak{a}_{v, \mu}-\mathfrak{a}_{\mu, v} .
$$


Eqs.(2.3) and (2.6) are invariant with respect to the gauge maps

$$
\begin{aligned}
h_{\mu v} & \mapsto h_{\mu v}-\xi_{\mu ; v}-\xi_{v ; \mu}, \\
\mathfrak{a}_{\mu} & \mapsto \mathfrak{a}_{\mu}+\phi_{; \mu}-\xi^{\alpha} A_{\mu ; \alpha}-\xi_{; \mu}^{\alpha} A_{\alpha},
\end{aligned}
$$

and the conditions $k_{; \beta}^{\alpha \beta}=0=\mathfrak{a}_{\alpha}^{\alpha}$ eliminate the arbitrariness of gauge maps. As proposed by Novikov et all [20] the geometrical optics approximations

$$
\begin{aligned}
\mathfrak{a}_{\mu} & =\Re\left(\mathfrak{b}_{\mu}+\varepsilon \mathfrak{c}_{\mu}+\cdots\right) \exp (i S / \varepsilon), \\
k_{\mu v} & =\Re\left(\kappa_{\mu \nu}+\varepsilon \pi_{\mu \nu}+\cdots\right) \exp (i S / \varepsilon)
\end{aligned}
$$

are used in Eqs.(2.3) and (2.6), and denoting $l_{\alpha}=S_{; \alpha}$, by setting the terms of order $\varepsilon^{-2}$ and $\varepsilon^{-1}$ equals zero, the following relations are obtained:

$$
\begin{aligned}
l_{\alpha} l^{\alpha} & =0, \quad l^{\mu} \kappa_{\mu v}=0=l^{\mu} \mathfrak{b}_{\mu}, \\
l_{; \beta}^{\beta} \mathfrak{b}^{\mu}+2 l^{\beta} \mathfrak{b}_{; \beta}^{\mu} & =l_{\beta}\left(\frac{1}{2} F^{\mu \beta} \kappa_{\alpha}^{\alpha}-F_{\gamma}{ }^{\beta} \kappa^{\gamma \mu}\right)
\end{aligned}
$$

These relations are analogous for the classic case proposed by Novikov [20], but when we incorporate braneworld effects, we have from Eq.(2.3) that

$$
l_{; \beta}^{\beta} \kappa_{\mu v}+2 l^{\beta} \kappa_{\mu v ; \beta}+2\left(\kappa^{; \beta} l_{\beta}+\kappa l_{; \beta}^{\beta}\right) g_{\mu v}=\tau_{\mu v},
$$

where

$$
\begin{aligned}
\tau_{\mu v}= & 2 g_{\mu \nu} \mathfrak{b}_{[\beta} l_{\alpha]}\left(F^{\alpha \beta} F^{2}-2 F_{\gamma}^{\beta} F^{\alpha \sigma} F_{\sigma}^{\gamma}\right)+\frac{F^{2}}{2}\left(\mathfrak{b}_{[v} l_{\alpha]} F_{\mu}{ }^{\alpha}-\mathfrak{b}_{[\mu} l_{\alpha]} F_{v}{ }^{\alpha}\right) \\
& +\mathfrak{b}_{[\beta} l_{\alpha]} F^{\gamma \alpha} F_{\gamma(\mu} F_{v)}^{\beta}-\left(\mathfrak{b}_{[\mu} l_{\alpha]} F_{\nu \beta}+\mathfrak{b}_{[v} l_{\alpha]} F_{\mu \beta} F^{\beta \gamma} F_{\gamma}{ }^{\alpha}+F_{\beta \alpha} F_{\gamma(v} F_{\mu)}^{\gamma}\right) .
\end{aligned}
$$

The first relation in Eq.(2.10) - the eikonal equation $l^{\alpha} l_{\alpha}=0-$ classifies the constant phase surface $S=$ constant is a null surface, and is characterized by null geodesics given by the integral lines $x^{\mu}=x^{\mu}(\lambda)$, defined by the equation $\frac{d x^{\mu}}{d \lambda}=l^{\mu}(x)$. A complex null tetrad $\left(l^{\mu}, n^{\mu}, m^{\mu}, \bar{m}^{\mu}\right)$ can be constructed in such a way that the unique non null scalar products are given by $l^{\mu} n_{v}=$ $-1, m^{\mu} \bar{m}_{\mu}=1$. Besides, they also satisfy $l^{\mu} m_{\mu}^{v}=l^{\mu} n_{\mu}^{v}=0=l^{\mu} m_{\mu}$, and by the choice

$$
\begin{aligned}
\mathfrak{b}_{\mu} & =A \bar{m}_{\mu}+\bar{A} m_{\mu}, \\
\kappa_{\mu v} & =2\left(H \bar{m}_{\mu} \bar{m}_{v}+\bar{H} m_{\mu} m_{v}\right)
\end{aligned}
$$

where $A=A(r)$ is the amplitude associated with the perturbation in the eletromagnetic potential, multiplying Eq.(2.11) by $m^{\mu}$ and Eq.(2.12) by $m^{\mu} m^{v}$ the following system of coupled equations is obtained:

$$
\begin{aligned}
& \frac{d A}{d \lambda}-\rho A=\bar{\phi}_{0} H \\
& \frac{d H}{d \lambda}-\rho H=-\phi_{0} A+\left[\Re(A) \phi_{0} F_{\alpha\left({ }_{v} F_{\mu}\right)}{ }^{\alpha}+h_{\mu v} F^{2}\right] m^{\mu} m^{v}
\end{aligned}
$$


where $\phi_{0}=F_{\mu \nu} l^{\mu} m^{v}$ and $\rho=-\frac{1}{2} l_{\alpha}^{\alpha}$. If we compare our results with the classical ones in [20], we see that there the system of equations

$$
\begin{gathered}
\frac{d A}{d \lambda}-\rho A=\bar{\phi}_{0} H \\
\frac{d H}{d \lambda}-\rho H=-\phi_{0} A
\end{gathered}
$$

come from Einstein-Maxwell equations for the 4D case, given by

$$
\begin{aligned}
& k_{\mu v ; \lambda}^{; \lambda}-k_{\mu ; v ; \lambda}^{\lambda}-k_{v ; \mu ; \lambda}^{\lambda}-\frac{1}{2} g_{\mu v} k_{; \lambda}^{; \lambda} \\
& -2 k^{\alpha \beta} F_{\mu \alpha} F_{v \beta}-\frac{1}{2} k_{\mu v} F^{2}+g_{\mu v} k^{\alpha \gamma} F_{\alpha \beta} F_{\gamma}{ }^{\beta}+k T_{\mu v}-2 F_{(\mu}^{\alpha} \delta F_{v) \alpha}+g_{\mu v} F^{\alpha \beta} \delta F_{\alpha \beta}=0
\end{aligned}
$$

together with Eq.(2.6). The term $\Re(A) \phi_{0} F_{\alpha\left({ }_{v}\right.} F_{\mu)}{ }^{\alpha}+h_{\mu v} F^{2}$ arises in fact by braneworld effects and can be used to detect possible extra-dimensional physical effects. For the case of a ReissnerNordstrøm BH, the brane-corrected metric is given by $g_{\mu \nu} d x^{\mu} \otimes d x^{v}=-H(r) d t \otimes d t+\frac{1}{H(r)} d r \otimes$ $d r+r^{2} d \Omega \otimes d \Omega$, where

$$
H(r)=1-\frac{2 G M}{c^{2} R_{\mathrm{RNbrane}}}+\frac{2 G \ell Q^{*}}{c^{2} R_{\mathrm{RNbrane}}}
$$

$Q^{*}$ denotes the bulk-induced tidal charge, and $\ell$ denotes the $\mathrm{AdS}_{5}$ bulk radius. Here $d \Omega \otimes d \Omega \equiv d \Omega^{2}$ denotes the 3-volume element related to the geometry of the 3-brane and $R_{\mathrm{RNb}}$-ane denotes the braneworld-corrected Reissner-Nordstrøm radius [15, 18, 19]

$$
R_{\mathrm{RNbrane}}=\frac{G M}{c^{2}}+\frac{1}{c}\left(\frac{G^{2} M^{2}}{c^{2}}-2 \ell G Q^{*}\right)^{1 / 2}
$$

It follows from Eq.(2.15) that the amplitude $A=A(r)$ associated with the perturbation in the electromagnetic potential satisfies the differential equation

$$
\left(\frac{d^{2}}{d r^{2}}+\frac{1}{r}\left(\frac{H(r)}{r^{2}}-1\right) \frac{d}{d r}+\frac{2 M}{r^{2} H(r)}+1-4 H(r)\right) A(r)=0 .
$$

This equation can be solved in a neighborhood excluding the singularity points, and for a wide range of radial values, we obtain different forms for the graphics, and some of them are depicted below. The scalar part $A$ associated with the perturbation of the electromagnetic field, given by $\delta A^{\mu}=\mathfrak{a}^{\mu}=A \bar{m}^{\mu}+\bar{A} m^{\mu}$ is transformed in the perturbation of gravitational field, associated with the black hole. Also, by numerical computational reasons there is only a little range where the solutions are 'well-behaved' - to be understood as the continuity of $A(r)$ up to third derivatives. 
(a)
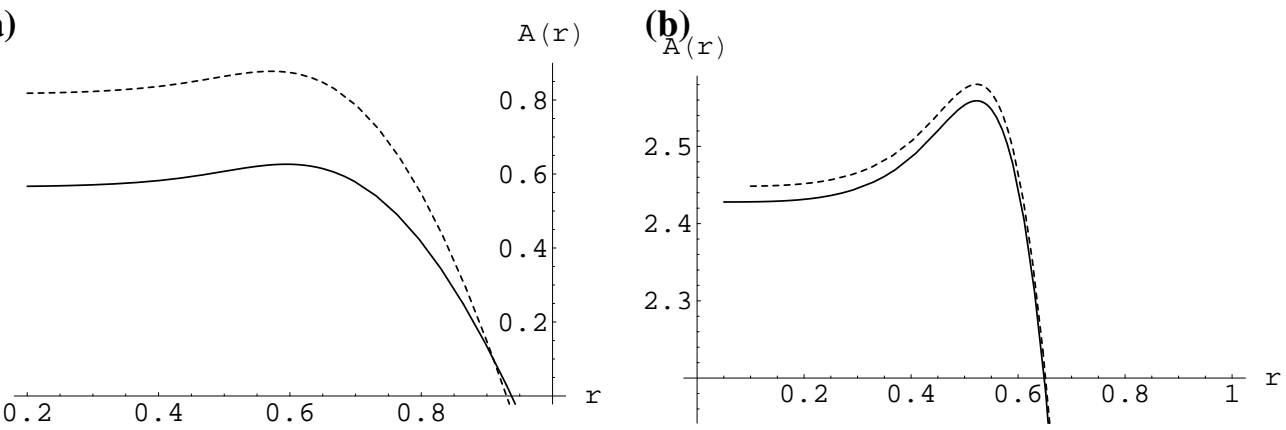

Figure 1: In both graphics, the dashed line indicates the braneworld-corrected amplitude of the EM potential perturbation, and the full line shows the amplitude of the EM potential perturbation in the Novikov's classical formalism framework. In graphic (a) the initial conditions are given by $A(0.91)=0.1, A^{\prime}(0.5)=0.3$, and in graphic (b) they are $A(0.82)=0.1, A^{\prime}(0.5)=0.3$

(c)

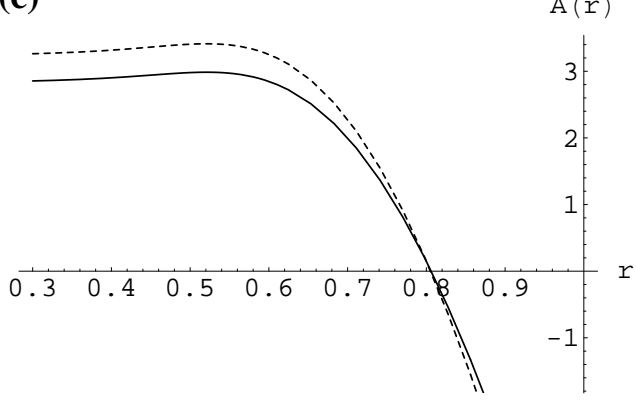

(d)

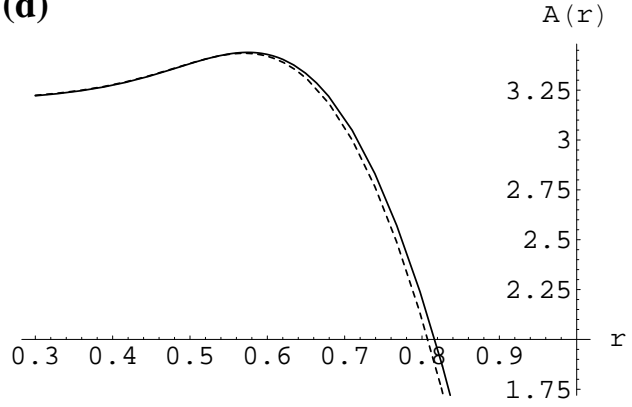

Figure 2: In both graphics, the dashed line indicates the braneworld-corrected amplitude of the EM potential perturbation, and the full line shows the amplitude of the EM potential perturbation in the Novikov's classical formalism framework. In graphic (c) the initial conditions are given by $A(0.91)=0.1, A^{\prime}(0.5)=0.3$, and in graphic (d) they are $A(0.82)=0.1, A^{\prime}(0.5)=0.3$

\section{Concluding remarks and outlooks}

We solved Eisntein-Maxwell equations on the brane and have shown how to obtain the braneworldcorrections of the perturbations in the EM potential around a Reissner-Nordstrøm black hole. These corrections are illustrated by the graphics in Figures 1 and 2. The system of coupled equations obtained in [20] has, in a Randall-Sundrum braneworld scenario, more terms involving only the amplitude of the perturbation in the EM potential, and the amplitude of perturbation in the metric of the 3-brane.

The system of equations given by Eqs.(2.16), implies the relation $\left[l^{\mu}\left(|A|^{2}+|H|^{2}\right)\right]_{; \mu}=0$, meaning that the total number of photons and gravitons is conserved. On the other hand, the branecorrected system of equations, given by Eqs.(2.15) does not conserve the total number of photons and gravitons on the brane, although it can be shown that this total number is in fact conserved on the brane plus the bulk. This last statement is a theorem involving the $\mathrm{AdS}_{5}$ bulk geometry and it is beyond the scope of the present paper to explicitly prove it. All these figures show, in 
accordance to [18], that the effective Reissner-Nordstrøm radius $R_{R N}$ is not determined by the usual classic equation $1-\frac{2 G M}{c^{2} R_{R N}}+\frac{Q^{2}}{c^{2} R_{R N}^{2}}=0$, but by the brane-corrected Reissner-Nordstrøm radius $R_{\text {RNbrane }}$ [18], given by Eq.(2.17). These corrections can also be achieved and generalized, using most general - e.g., Kerr-Newman — black holes and branes possessing different codimension [24]. Also, the relationship between mutual transformations of EM and gravitational fields can be described and investigated in terms of quasinormal modes $[12,25]$.

\section{References}

[1] Green M B, Schwarz J H, and Witten E, Superstring Theory, vols. I \& II, Cambridge Univ. Press, Cambridge 1987.

[2] Dienes K R, String theory and the path to unification: a review of recent developments, Phys. Rep. 287, 447-525 (1997) [hep-th/9602045].

[3] Kaku M, Strings, Conformal Fields and M-theory, Springer-Verlag, New York 2000.

[4] Kiritsis E, Introduction to Superstring Theory, Leuven Notes in Math. and Theor. Phys. 9, Leuven Univ. Press, Leuven 1997 [hep-ph/9709062].

[5] Randall L and Sundrum R, An alternative to compactification, Phys. Rev. Lett. 83 4690-4693 (1999) [hep-th/9906064].

[6] Randall L and Sundrum R, A large mass hierarchy from a small extra dimension, Phys. Rev. Lett. 83, 3370- 3373 (1999) [hep-ph/9905221].

[7] Koyama $\mathrm{K}$ and Mizuno S, Inflaton perturbations in brane-world cosmology with induced gravity, JCAP, 0713 (2006) 013.

[8] Cooperstock F I, The interaction between electromagnetic and gravitational waves, Ann. Phys. 47, 173-181 (1968).

[9] Alekseev G A and Sibgatullin N R, Effect of the mutual conversion of electromagnetic and gravitational waves in strong external electromagnetic fields and wave propagation in the field of a charged 'black hole', Prikladnaia Matematika i Mekhanika 38, 1122-1129 (1974).

[10] Gerlach U H, Beat frequency oscillations near charged black holes and other electrovacuum geometries, Phys. Rev. Lett. 32, 1023-1025 (1974).

[11] Marklund M, Brodin G, and Dunsby P K S, Radio wave emissions due to gravitational radiation, Astrophys. J. 536, 875-879 (2000) [astro-ph/9907350].

[12] Clarkson C, Marklund M, Betschart G, and Dunsby P, The electromagnetic signature of black hole ring-own, Astrophys. J. 613, 492-505 (2004) [astro-ph / 0310323 ].

[13] Marklund M, Dunsby P K S, and Brodin G, Cosmological electromagnetic fields due to gravitational wave pertubations, Phys. Rev. D62 (2000) 101501 [gr-qc/ 0007035 ].

[14] Randall L and Sundrum R, An alternative to compactification, Phys. Rev. Lett. 83, 4690-4693 (1999) [hep-th/9906064].

[15] Maartens R, Brane-World Gravity, Living Rev. Rel. 7,7 (2004) [qr-qc/ 0312059 ].

[16] Easther R, Langlois D, Maartens R, and Wands D, Evolution of gravitational waves in Randall-Sundrum cosmology, JCAP 0310 (2003) 014 [hep-th / 0308078 ]. 
[17] Coimbra-Araújo C H, da Rocha R, and Pedron I T, Anti-de Sitter curvature radius constrained by quasars in brane-world scenarios, Int. J. Mod. Phys. D14 (2005) 1883-1898

[astro-ph/0505132].

[18] da Rocha R and Coimbra-Araújo C H, Variation in the luminosity of Kerr quasars due to an extra dimension in the brane Randall-Sundrum model, JCAP 0512 (2005) 009 [astro-ph / 0510318 , astro-ph/0509363].

[19] da Rocha R and Coimbra-Araújo C H, Extra dimensions in LHC via mini-black holes: effective Kerr-Newman brane-world effects, Phys. Rev D74 (2006) 055006 [hep-ph / 0607027 ].

[20] Novikov D and Frolov V P, Physics of Black Holes, Kluwer, Dordrecht 1989.

[21] Seahra S S, Clarkson C, and Maartens R, Detecting extra dimensions with gravity wave spectroscopy: the black string brane-world, Phys. Rev. Lett. 94 (2005) 121302 [gr-qc/ 0408032 ].

[22] Wald R M, General Relativity, University of Chicago Press, Chicago 1984.

[23] Shiromizu T, Maeda K, and Sasaki M, The Einstein equations on the 3-brane world, Phys. Rev. D62 (2000) 024012-1-6 [gr-qc/9910076].

[24] de Rham C and Tolley A J, Gravitational waves in a codimension two braneworld, JCAP 0206 (2006) 003 [hep-th/0511138].

[25] da Rocha R and Capelas de Oliveira E, Conformal Klein-Gordon equations and quasinormal modes, Int. J. Theor. Phys. (2006) DOI: 10.1007/s10773-006-9238-5 [gr-qc/ 0603082 ]. 